\title{
The Combination of Serum Cystatin C, Urinary Kidney Injury Molecule-1 and MELD plus Score Predicts Early Acute Kidney Injury after Liver Transplantation
}

\author{
Marian-Irinel Tudoroiu ${ }^{1}$, Georgiana Constantin ${ }^{1}$, Liliana Pâslaru ${ }^{1,2}$, Speranța lacob ${ }^{1,2}$, \\ Cristian Gheorghe ${ }^{1,2}$, Irinel Popescu ${ }^{1}$, Dana Tomescu ${ }^{1,2}$, Liliana Simona Gheorghe ${ }^{1,2}$
}

'Fundeni Clinical Institute, Bucharest, Romania

${ }^{2 " C a r o l ~ D a v i l a " ~ U n i v e r s i t y ~ o f ~ M e d i c i n e ~ a n d ~ P h a r m a c y, ~ B u c h a r e s t, ~ R o m a n i a ~}$

\section{ABSTRACT}

Introduction: Acute kidney injury (AKI) following liver transplantation (LT) is a frequent complication and is associated with increased morbidity and mortality.

Aim: To investigate whether the levels of urinary KIM-1 and serum Cystatin $\mathrm{C}$ are able to predict early occurrence (within the first 48 hours) of the post-LT renal dysfunction.

Methods: The study was conducted on 25 recipients transplanted in the Fundeni Clinical Institute between May 2016 and February 2017. Serum Cystatin C, urinary KIM-1 and serum creatinine were analysed before LT, as well as 4 and 24 hours after graft reperfusion. In defining renal failure, the criteria of The Acute Kidney Injury Network (AKIN) were used. All patients received the same renal sparing regimen of immunosuppression (basiliximab, mycophenolate mofetil and delayed tacrolimus).

Results: Ten patients $(40 \%)$ had early post-LT $(<48 \mathrm{~h})$ renal dysfunction according to the AKIN classification. In the AKI group, there was a considerable increase of serum Cystatin $C$ at 4 and $24 \mathrm{~h}$ after LT in comparison with baseline values $(p=0.011)$, whereas in the group without AKI, the values of Cystatin $C$ dropped $(p=0.11)$. There were no significant differences between the KIM-1 values between the two groups. The single independent risk factor for early post-LT AKI occurrence was serum Cystatin $C$ value 4 hours after hepatic reperfusion. The clinical utility of serum Cystatin $\mathrm{C}$ and urinary KIM-1 $4 \mathrm{~h}$ after reperfusion, evaluated by AUROC, was good (0.79). However, the combination of the two biomarkers and the MELD plus score had a better performance, with an AUROC of 0.83 , an $80 \%$ sensitivity and $80 \%$ specificity for distinguishing patients with early post-transplant AKI from those without AKI.

Conclusion: Early renal dysfunction following LT is frequent (40\%) despite using a renal sparing immunosuppression regimen. Combination of serum Cystatin $C$ and urinary KIM-1, 4 hours after hepatic reperfusion and pre-LT MELD-plus score performed the best in screening the early post-LT renal dysfunction.

Key words: biomarkers, acute kidney injury, liver transplantation, kidney injury molecule-1, cystatin C

\section{INTRODUCTION}

Acute kidney injury (AKI) after liver transplantation (LT) is a frequent complication and is associated with an increase in morbidity, mortality and
Corresponding author: Speranța lacob, MD

Fundeni Clinical Institute, Bucharest Romania

E-mail:msiacob@gmail.com

\section{Abbreviations:}

AKI - Acute Kidney Injury;

AKIN - Acute Kidney Injury Network: AUROC - Area Under the Receiver Operating Characteristic curve; LT - Liver Transplantation; MELD - Model for End Stage Liver Disease;

Received: 3.03.2018

Accepted: 4.04.2018

Copyright (1) Celsius Publishing House www.sgo-iasgo.com 
hospitalisation time. Depending on the criteria used in defining AKI, the incidence ranged between 17 and 95\% (1). Patients with a low glomerular filtration rate within a month after the LT have an increased risk of developing severe kidney failure within 5 years following $\mathrm{LT}$; the need dialysis in LT recipients within 5 years is $18 \%(2,3)$.

Since the introduction of the Model for End Stage Liver Disease (MELD) for the allocation of organs for LT in 2002, the inclusion of serum creatinine in the MELD score has significantly increased the incidence of renal dysfunction seen among patients undergoing LT. In 2005, Ruf et al (4) demonstrated that hyponatremia appears to be an earlier and more sensitive marker than serum creatinine able to detect renal impairment and/or circulatory dysfunction in patients with advanced cirrhosis. A more recent score, MELD-Plus (including all MELD-Na components, as well as albumin, total cholesterol, white blood cell count, age, length of stay in hospital) resulted in an improvement over MELD and MELD-Na scores in predicting short-term mortality of patients with established cirrhosis (5).

Multiple factors can be involved in the early detection of post-transplant AKI, such as: pretransplant kidney dysfunction, severe hepatic disease, hypoalbuminemia, the duration of the intraoperative vascular clamping (which interferes with the renal flow), cold ischemia time, the amount of intraoperative transfusion, perioperative hypotension and the necessary amount of vasopressor support, the duration of the surgery, and the nephrotoxic medication such as calcineurin inhibitors (6-13). Although serum creatinine level remains the most common test in assessing the renal function, there are multiple drawbacks associated to it: it is influenced by diet, muscle mass, age and gender; it has low sensitivity to small changes of the renal function, as well as a considerable delay from kidney injury until its increase. This delay in diagnosing AKI implies missing a therapeutic opportunity, the failure to correct certain triggering factors (13). In trying to identify an early marker of the renal dysfunction, which is not influenced by muscle mass, diet, gender and race, multiple markers were studied, and some of them proved to have clinical utility (14-16). Cystatin C is a non-glicolized protein with small molecular weight $(13.3 \mathrm{kDa})$, a hundred times bigger than creatinine. It is produced at a constant rate by all the nucleated cells, is freely filtered by glomeruli and minimally linked to proteins, and is not reabsorbed in the systemic circulation after the filtering. KIM-1 is a transmembrane glycoprotein whose expression at the level of the cells of the proximal renal tubule is increased in case of ischaemia and nephrotoxic substances. An extracellular domain of KIM-1, of $90 \mathrm{kDa}$, is detectable in the urine immediately after kidney injury $(17,18)$. KIM-1 is a promising biomarker for the diagnosis and the prognosis of AKI (19-21).

The purpose of the study was to investigate urinary KIM-1 and serum Cystatin $C$ in detecting the early occurrence (within the first 48 hours) of the post-LT renal dysfunction.

\section{METHOD}

The study was conducted on 25 liver transplant recipients in Fundeni Clinical Institute between May 2016 and February 2017. Exclusion criteria consisted of paediatric patients, pre-existing renal impairment and refusal to consent. All patients in the study group received a renal sparing immunosuppressive regimen comprised of basiliximab, tacrolimus and mycophenolate mofetil, as per institutional protocol.

An analysis was conducted on their demographical data (age, gender, body mass index), lab reports (before the $L T$, in the intra-operative stage, and on 4 consecutive days after the LT). Before the LT, 4 and 24 hours after the hepatic reperfusion, serum Cystatin C, urinary KIM-1 and serum creatinine were analysed. The following intraoperative data was also recorded: requirement of blood products, the duration of the surgery, cold ischemia time, warm ischemia time, the presence or absence of the reperfusion syndrome, hemodynamic parameters (PICCO monitoring), the amount of vaso-pressor support. Serum Cystatin $C$ and urinary KIM-1 were measured by the ELISA method. The patients were split into two groups depending on the occurrence of the early renal dysfunction ( $<48$ hours): with and without AKI. In defining renal failure, the criteria of The Acute Kidney Injury Network (AKIN) were used - see table $1(22,23)$.

\section{Statistical analysis}

The demographic data about the 2 groups were subjected to a comparative analysis, with or without AKI, by using Fisher's exact test or Student $t$ test. Depending on the data that were analysed, the average and the standard deviation or the median and the interval of the values were calculated. The means of the pre-transplant parameters were compared with the post-surgery ones by using $t$ test paired or $t$ test unpaired, on a case by case basis. The ANOVA test was used to determine if the differences between the means of several sets of numerical data were statistically significant $(p<0.05)$. To determine if the 


\section{Table 1 - The Acute Kidney Injury Network (AKIN) classification*}

Serum creatinine criteria:

\begin{tabular}{|c|c|c|}
\hline Stage & 1 & $\begin{array}{l}\text { Increase in serum creatinine } \geq 0.3 \mathrm{mg} / \mathrm{dl} \text { ( } \geq 26.4 \mu \mathrm{mol} / \mathrm{l}) \text { or increase to } \geq 150 \% \text { to } 200 \% \text { ( } 1.5 \text { to } 2 \text {-fold }) \\
\text { from baseline }\end{array}$ \\
\hline & $2^{\&}$ & Increase in serum creatinine to more than $200 \%$ to $300 \%$ ( $>2$ to 3 -fold) from baseline \\
\hline & $3^{\#}$ & $\begin{array}{l}\text { Increase in serum creatinine to more than } 300 \%(>3 \text { fold }) \text { from baseline (or serum creatinine } \geq 4.0 \mathrm{mg} / \mathrm{dl} \\
[\geq 354 \mu \mathrm{mol} / \mathrm{l}] \text { with an acute increase of at least } 0.5 \mathrm{mg} / \mathrm{dl}[44 \mu \mathrm{mol} / \mathrm{l}])\end{array}$ \\
\hline Urine & ut $\mathrm{c}$ & \\
\hline Stage & 1 & Less than $0.5 \mathrm{ml} / \mathrm{kg}$ per hour for more than 6 hours. \\
\hline & 2 & Less than $0.5 \mathrm{ml} / \mathrm{kg}$ per hour for more than 12 hours \\
\hline & 3 & Less than $0.3 \mathrm{ml} / \mathrm{kg}$ per hour for 24 hours or anuria for 12 hours \\
\hline
\end{tabular}

${ }^{*}$ Modified from RIFLE (Risk, Injury, Failure, Loss, and End-stage kidney disease) criteria (23). The staging system proposed is a highly sensitive interim staging system and is based on recent data indicating that a small change in serum creatinine influences outcome. Only one criterion (creatinine or urine output) has to be fulfilled to qualify for a stage.

${ }^{2} 200 \%$ to $300 \%$ increase $=2$ - to 3 -fold increase

"Given wide variation in indications and timing of initiation of renal replacement therapy (RRT), individuals who receive RRT are considered to have met the criteria for stage 3 irrespective of the stage they are in at the time of RRT.

differences between the medians of two sets of data were statistically significant, Mann-Whitney $U$ test was used. The statistically significant parameters in the univariate analysis were analysed through multivariate logistic regression. The AUROC (Area Under the Receiver Operating Characteristic curve) was used to determine the best cut off value of the analysed parameters that predict post-LT AKI. The tests were considered statistically significant if $p<0.05$.

\section{RESULTS}

The study included $36 \%$ females and $64 \%$ males, with a mean age of $50.56( \pm 10.28)$, MELD score 18.52
Table 2 - Baseline characteristics of the study group

\begin{tabular}{lc}
\hline Age (years) & $50.56 \pm 10.28$ \\
\hdashline Gender (males) & $60 \%$ \\
\hline MELD & $18.52( \pm 6.56)$ \\
\hline MELD PLUS & $0.13(0.01-0.72)$ \\
\hline Total Bilirubin $(\mathrm{mg} / \mathrm{dl})$ & $3(0.2-24.1)$ \\
\hline INR & $1.65( \pm 0.42)$ \\
\hline Creatinine $(\mathrm{mg} / \mathrm{dl})$ & $0.85( \pm 0.18)$ \\
\hline Albumin $(\mathrm{mg} / \mathrm{dl})$ & $3.31( \pm 0.66)$ \\
\hdashline Na $(\mathrm{mmol} / \mathrm{l})$ & $135.8( \pm 5.52)$ \\
\hline Cystatin C $(\mathrm{ng} / \mathrm{ml})$ & $1210( \pm 504.7)$ \\
\hdashline KIM-1(ng/ml) & $0.156(0.02-5.82)$ \\
\hline
\end{tabular}

Table 3 - The risk factors analysed for the occurrence of the post-LT AKI in the two groups

\begin{tabular}{|c|c|c|c|}
\hline & With AKI(n=10) & Without AKI(n=15) & p value \\
\hline Age (years) & $53.7( \pm 5.65)$ & $48.47( \pm 12.21)$ & 0.21 \\
\hline Gender (males) & $7(70 \%)$ & $9(60 \%)$ & 0.62 \\
\hline MELD & $20.4( \pm 5.14)$ & $17.27( \pm 7.25)$ & 0.25 \\
\hline MELED PLÜS & $0.14(0.03-0.72)$ & $0.08(0.01-0.49)$ & 0.25 \\
\hline Total Bilirubin (mg/dl) & $2.8(1.3-24.1)$ & $3.9(0.2-19.3)$ & 0.95 \\
\hline INR & $1.68( \pm 0.46)$ & $1.64( \pm 0.4)$ & 0.78 \\
\hline Baseline creatinine & $0.86( \pm 0.21)$ & $0.84( \pm 0.17)$ & 0.75 \\
\hline Albumin (mg/dl) & $2.98( \pm 0.61)$ & $3.53(2.7-4.6)$ & $0.030^{\star}$ \\
\hline $\mathrm{Na}(\mathrm{mmol} / \mathrm{l})$ & $134.8( \pm 4.56)$ & $136.4( \pm 6.15)$ & 0.48 \\
\hline Baseline Cystatin (ng/ml) & $1216( \pm 630.3)$ & $1205( \pm 425.7)$ & 0.95 \\
\hline Cystatin (ng/ml) -4h & $1203( \pm 338.6)$ & $852( \pm 310.1)$ & $0.013^{*}$ \\
\hline Baseline KIM-1 (ng/ml) & $0.6(0.02-3.94)$ & $0.14(0.03-5.82)$ & 0.28 \\
\hline $\mathrm{K} I \mathrm{IM}-1(\mathrm{ng} / \mathrm{ml})-4 \mathrm{~h}$ & $0.71(0.07-1.81)$ & $0.26(0.06-2.45)$ & 0.6 \\
\hline Bleeding $(L)$ & $5.25( \pm 2.89)$ & $4.73( \pm 4.35)$ & 0.74 \\
\hline Cold ischemia time (min) & $342.8( \pm 126)$ & $256.1( \pm 91.71)$ & 0.07 \\
\hline Warm ischemia time (min) & $42.75( \pm 17.47)$ & $41.43( \pm 15.25)$ & 0.85 \\
\hline Duration of the LT (min) & $416( \pm 135.3)$ & $410( \pm 88.48)$ & 0.89 \\
\hline
\end{tabular}


( \pm 6.56$)$, and with MELD plus $0.13(0.01-0.72)$ - see table 2. The aetiology of hepatic cirrhosis in the analysed cohort was: HBV related cirrhosis - 15 (60\%), 6 (24\%) patients had HCV related cirrhosis, 3 (12\%) alcoholic cirrhosis and 4\% other causes; 6 (24\%) also had associated hepatocellular carcinoma.

In an early post-LT phase $(<48 \mathrm{~h}), 10$ patients $(40 \%)$ had renal dysfunction according to the AKIN classification. table 3 presents the risk factors analysed for the occurrence of the post-LT AKI.

There were no statistically significant differences between the two groups regarding the demographic data and the analysed parameters, except for a lower value of serum albumin in the AKI group $(p=0.03)$ (11). There was a statistically significant difference between the values of Cystatin $C$ within 4 and 24 hours after the hepatic reperfusion between the two groups $(p=0.013$ and $p=0.001$, respectively). In the AKI group, there was a considerable increase in comparison with baseline values $(p=0.011)$, whereas in the group without AKI, the values of Cystatin C dropped ( $p=0.11)$ - see fig. 1 .

There were no significant differences between KIM-1 values in the two groups in neither before nor after graft implantation - see fig. 2 .

The analysis of multivariate logistic regression found that the only independent risk factor for the post-LT occurrence of AKI was the value of Cystatin 4 hours after the hepatic reperfusion. A lower value of serum albumin before surgery is a risk factor for early post-LT AKI, but with marginal statistical significance $(p=0.056)$.

The value of serum Cystatin $C$ within 4 hours showed a considerable discriminatory ability to distinguish patients who had early post-LT renal dysfunction $(<48$ hours) from those who did not have it (AUROC of 0.747, $p=0.04$ ) - see fig. 3. The corresponding cut off for Cystatin C is $1202.97 \mathrm{ng} / \mathrm{ml}$ with $50 \%$ sensitivity, but with 93.33\% specificity in diagnosing AKI in liver-transplanted patients. Pre-LT MELD plus score plays a predictive role in the occurrence of post-LT AKI with AUROC $0.64(p=0.24)$ for a cut off $>0.25$, with $30 \%$ sensitivity, but very high specificity, namely $93.33 \%$. The combination of the two biomarkers, Cystatin C and KIM-1 4 hours after hepatic reperfusion, had AUROC of 0.79 with $70 \%$ sensitivity and $80 \%$ specificity for the diagnosis of AKI in livertransplanted patients. The clinical utility of the pattern

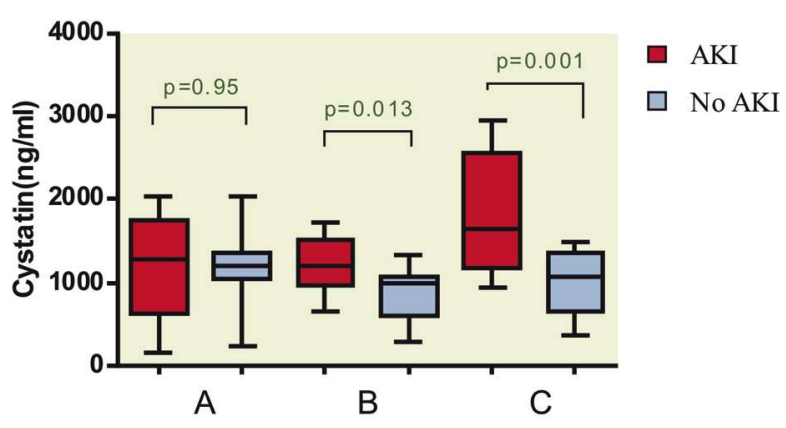

Figure 1 - Cystatin C (ng/ml) in pre-LT (A), 4 hours after hepatic reperfusion (B) and 24 hours after hepatic reperfusion (C) in the AKI group in comparison with the group without AKI

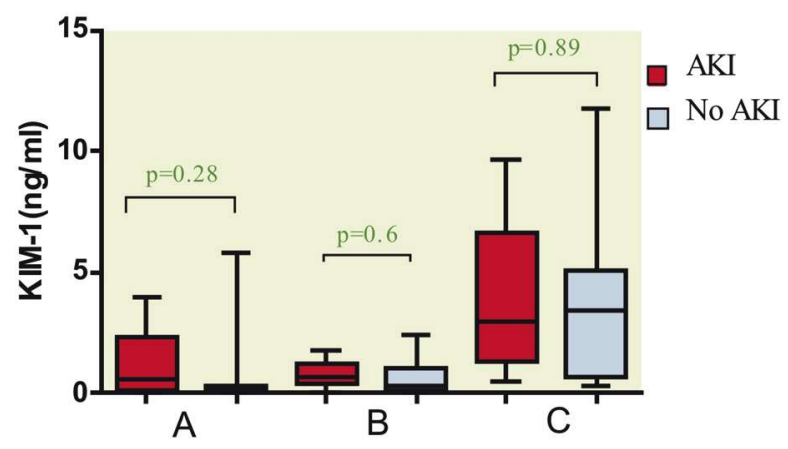

Figure 2 - KIM-1 (ng/ml) in pre-LT (A), 4 hours after hepatic reperfusion (B), and 24 hours after hepatic reperfusion (C) in the AKI group in comparison with the group without AKI.

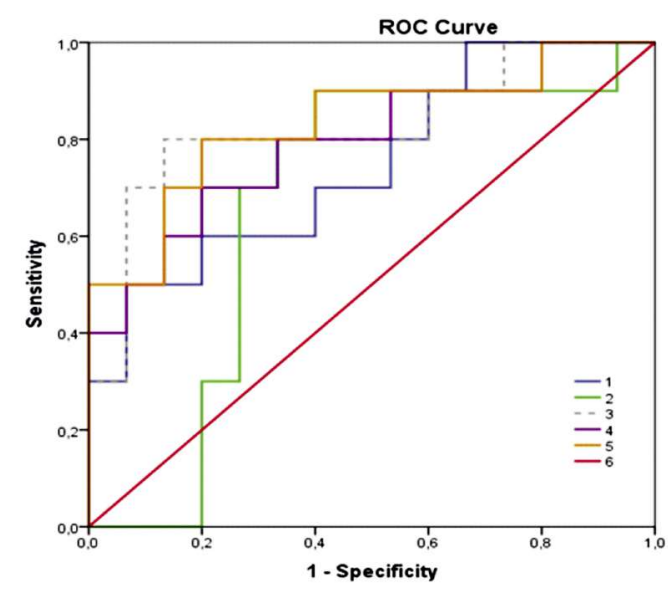

Figure 3

Legend: 1 = AUROC for Cystatin C; 2 = AUROC for KIM-1; $3=$ AUROC for KIM + Cystatin C + MELD score $4=$ AUROC for KIM-1 + Cystatin C $\mathbf{5}=$ AUROC for KIM + Cystatin + MELD-PLUS score

Table 4 - Results of the multivariate logistic regression analysis

\begin{tabular}{lccc}
\hline Variable & Regression coefficient & Std error & P value \\
\hline Cystatin C 4 hours after LT & 0.004 & 0.002 & 0.036 \\
\hline Pre-LT Serum albumin & -1.56 & 0.82 & 0.056 \\
\hline
\end{tabular}


Table 5 - AUROC values for predicting early AKI after LT

\begin{tabular}{lcc}
\hline Test Result Variable(s) & Area & $\mathbf{p}$ \\
\hline CYSTATIN_C_4hours & 0.747 & 0.040 \\
\hline KIM_1_4hours & 0.667 & 0.166 \\
\hdashline CYSTATIN_C_4hours+KIM_1_4hours+MELD & 0.827 & 0.007 \\
\hdashline CYSTATIN_C_4hours+KIM_1_4hours & 0.793 & 0.015 \\
\hdashline CYSTATIN_C_4hours+KIM_1_4hours+MELD_PLUS & 0.833 & 0.006 \\
\hline
\end{tabular}

containing the two biomarkers and MELD plus score was higher - AUROC of 0.83 ( $p=0.006), 80 \%$ sensitivity, and $80 \%$ specificity - in distinguishing patients with AKI from those without AKI. This combination of Cystatin $C$ and KIM-1 4 hours after hepatic reperfusion and pre-LT MELD plus score performed the best in screening the early post-LT renal dysfunction - see fig. 3.

\section{DISCUSSION}

Acute renal failure after LT is associated with short, as well as long term higher morbidity and mortality. However, early therapeutic or preventive intervention is hampered by the lack of early effective prognostic factors.

Many studies have attempted to determine risk factors for the development of post-LT renal dysfunction. Factors such as preoperative serum creatinine, early severe graft dysfunction consequent to use of marginal grafts, postoperative sepsis, low preoperative albumin and hepatic ischaemia reperfusion injury have all been implicated in the development of renal dysfunction after LT $(6,12,24)$. In our study, a lower level of preimplantation serum albumin was reported in the post-LT AKI group, similar to the study of findings reported by Cabezuelo et al (6). Hypoalbuminemia may increase the likelihood of AKI, since it reduces the glomerular filtration rate and alters the pharmacokinetics of potentially nephrotoxic drugs. Pre-transplant renal dysfunction, as well as more advanced liver insufficiency before LT, as reflected in a higher MELD score, predicted AKI after LT. In our study, MELD score did not perform well for predicting AKI after LT (AUROC of 0.57). However, MELD plus, a new nine variable score (including more risk factors for AKI, such as hypoalbuminemia and MELD-Na score), performed better for prediction of AKI after LT. To the best of our knowledge, this is the first study to assess MELD-plus score as a risk factor for post-LT AKI

Although a renal sparing immunosuppression protocol is used in our Transplant Centre (tacrolimus is introduced in the 5th postoperative day), AKI occurred early after LT in almost half of the analysed patients. Cystatin $\mathrm{C}$ is a ubiquitous protein that is freely filtered by the kidney and metabolised by the tubules. KIM-1 is a transmembrane protein that is expressed minimally in normal kidneys, but up-regulated dramatically in injured kidneys (25).

Cystatin C-based equations may be more accurate indicators of glomerular filtration rate than creatininebased equations, as previously demonstrated both in cirrhotic patients and after LT. A cystatin C-based MELD score was proposed, but it did not improve the predictive power of MELD on the waiting list (26). In contrast, in our study, combined Cystatin C and MELD or MELD-plus scores showed a better prediction of post-LT AKI compared to MELD alone. In the study of Hei et al (27), Cystatin C before LT as well as at the end of the operation correlated with early acute renal failure. Unlike the study conducted by Sirota et al (28), in our study there were statistically significant differences between the values of Cystatin $C$ in the two groups, i.e. 4 and 24 hours after hepatic reperfusion.

In our study, serum Cystatin C 4 hours after hepatic reperfusion had a substantially higher value than the value of urinary KIM-1 within 4 hours in predicting the occurrence of an early post-LT AKI. A combination of these two biomarkers was superior to each marker individually. The best pattern in predicting an early post-LT AKI proved to be the one combining the 2 biomarkers and the MELD plus score. KIM-1 is a potential novel urinary biomarker in the early detection of AKI within 24 hours after kidney injury, and it might be especially beneficial in the diagnosis of ischaemic acute tubular necrosis. KIM-1 alone within 4 hours after LT did not achieve a good prognostic value for predicting AKI in our study. This is similar to a recent study showing a low AUROC for both serum and urinary KIM-1 in diagnosing severe acute tubular injury, or any acute tubular injury for that matter (29). The low predictive value of KIM-1 for AKI after LT in our cohort demonstrates a good management of the transplanted patients during the surgery procedure, without hemodynamic and hypovolemic changes.

The limitations of our study are the small sample, the short follow up period for AKI. However, this is the first post-transplant study to analyse biomarkers for kidney dysfunction after LT in Romania.

\section{CONCLUSIONS}

The purpose of the study was attained by creating a pattern consisting of the value of MELD plus, serum 
Cystatin C 4 hours after hepatic reperfusion, and urinary KIM-1 within 4 hours, capable of accurately predicting an AKI early, in the first 48 hours after a liver transplantation. This model is highly useful, given the frequency of the post-LT AKI, the costs, and the comorbidities that stem from it. A superior value of this pattern is given by the inability to predict early AKI by the other analysed factors. In the group of patients who were analysed, the pattern that was identified predicted AKI at least 16 hours prior to its occurrence, thus enabling a therapeutic window in which the triggering factors could be corrected.

\section{Conflict of interests}

The authors declare no conflict of interests.

\section{REFERENCES}

1. Barri YM, Sánchez EQ, Jennings LW, Melton LB, Hays S, Levy MF, et al. Acute kidney injury following liver transplantation: Definition and outcome. Liver Transpl. 2009;15(5):475-83. doi: 10.1002/tt.21682.

2. Herlenius G, Fistouris J, Olausson M, Felldin M, Bäckman L, Friman $\mathrm{S}$. Early renal function post-liver transplantation is predictive of progressive chronic kidney disease. Scand J Gastroenterol. 2008 43(3):344-9.

3. Ojo AO, Held PJ, Port FK, Wolfe RA, Leichtman AB, Young EW, et al. Chronic renal failure after transplantation of a nonrenal organ. N Engl J Med. 2003;349(10):931-40.

4. Ruf AE, Kremers WK, Chavez LL, Descalzi VI, Podesta LG, Villamil FG. Addition of serum sodium into the MELD score predicts waiting list mortality better than MELD alone. Liver Transpl. 2005;11(3): 336-43.

5. Kartoun U, Corey K, Simon T, Zheng H, Aggarwal R, Ng K, et al. The MELD-Plus: A generalizable prediction risk score in cirrhosis Published: October 25, 2017. https://doi.org/10.1371/journal. pone. 0186301 .

6. Cabezuelo JB, Ramírez P, Ríos A, Acosta F, Torres D, Sansano T, et al. Risk factors of acute renal failure after liver transplantation. Kidney Int. 2006;69(6):1073-80.

7. Lima EQ, Zanetta DM, Castro I, Massarollo PC, Mies S, Machado MM, et al. Risk factors for development of acute renal failure after liver transplantation. Ren Fail. 2003;25(4):553-60.

8. Yalavarthy R, Edelstein CL, Teitelbaum I. Acute renal failure and chronic kidney disease following liver transplantation. Hemodial Int 2007, 11 (Suppl 3): S7-S12.

9. Yang GZ, Xue FS, Li HX. Assessing risk factors of acute kidney injury after liver transplantation. J Cancer Res Clin Oncol. 2017;143(12): 2635-2636. doi: 10.1007/s00432-017-2492-5. Epub 2017 Aug 17.

10. Hilmi IA, Damian D, Al-Khafaji A, Planinsic P, Boucek C, Sakai T, et al. Acute kidney injury following orthotopic liver transplantation: incidence, risk factors, and effects on patient and graft outcomes. $\mathrm{Br}$ J Anaesth. 2015;114(6):919-26. doi: 10.1093/bja/aeu556. Epub 2015 Feb 10.

11. Wiedermann CJ, Wiedermann W, Joannidis M. Hypoalbuminemia and acute kidney injury: a meta-analysis of observational clinical studies. Intensive Care Med. 2010;36(10):1657-65.

12. Suehana Rahman, Brian R Davidson, Susan V Mallett. Early acute kidney injury after liver transplantation: Predisposing factors and clinical implications. World J Hepatol 2017 June 28; 9(18): 823832.

13. Angeli P, Bezinover D, Biancofiore G, Bienholz A, Findlay J, Paugam
BC, Reyntjens K, Sakai T, Saner FH, Tomescu D, Wagener G, Weiss E. Acute kidney injury in liver transplant candidates: a position paper on behalf of the Liver Intensive Care Group of Europe. Minerva Anestesiol 2017;83(1):88-101.

14. Li Y, Zhu M, Xia Q, Wand WS, Qian J, Lu R, et al. Urinary neutrophil gelatinase-associated lipocalin (NGAL) and L-type fatty acid-binding protein (L-FABP) as diagnostic markers of early acute kidney injury after liver transplantation. Biomarker 2012;17(4):336-342.

15. Martensson J, Martling CR, Bell M. Novel biomarkers of acute kidney injury and failure: clinical applicability. British Journal of Anaesthesia 2012; 109 (6): 843-50.

16. Markwardt D, Holdt L, Steib C, Benesic A, Bendtsen F, Bernardi M, et al. Plasma cystatin $C$ is a predictor of renal dysfunction, acute-onchronic liver failure, and mortality in patients with acutely decompensated liver cirrhosis. Hepatology. 2017; 66(4):1232-1241.

17. Ichimura T, Bonventre JV, Bailly V, Wei H, Hession CA, Cate RL, et al. Kidney Injury Molecule-1 (KIM-1), a putative epithelial cell adhesion molecule containing a novel immunoglobulin domain, is up-regulated in renal cells after injury. J Biol Chem. 1998;273(7):4135-42.

18. Vaidya VS, Ramirez V, Ichimura T, Bobadilla NA, Bonventre JV. Urinary kidney injury molecule-1: a sensitive quantitative biomarker for early detection of kidney tubular injury. Am J Physiol Renal Physiol. 2006;290(2):F517-29. Epub 2005 Sep 20.

19. Liangos 0 , Perianayagam MC, Vaidya VS, Han WK, Wald R, Tighiouart $\mathrm{H}$, et al. Urinary $\mathrm{N}$-acetyl-beta-(D)-glucosaminidase activity and kidney injury molecule-1 level are associated with adverse outcomes in acute renal failure. J Am Soc Nephrol. 2007; 18(3):904-12. Epub 2007 Jan 31

20. Han WK, Waikar SS, Johnson A, Betensky RA, Dent CL, Devarajan $P$, et al. Urinary biomarkers in the early diagnosis of acute kidney injury. Kidney Int. 2008;73(7):863-9. Epub 2007 Dec 5. Erratum in Kidney Int. 2009;76(3):348-9.

21. Liangos 0 , Tighiouart $H$, Perianayagam MC, Kolyada A, Han WK, Wald $\mathrm{R}$, et al. Comparative analysis of urinary biomarkers for early detection of acute kidney injury following cardiopulmonary bypass. Biomarkers. 2009; 14(6):423-31.

22. Mehta RL, Kellum JA, Shah SV, Molitoris BA, Ronco C, Warnock $D G$, et al. Acute Kidney Injury Network: report of an initiative to improve outcomes in acute kidney injury. Critical Care 2007; 11(2):R31.

23. American Society of Nephrology Renal Research Report. J Am Soc Nephrol. 2005:16:1886-1903. doi: 10.1681/ASN.2005030285.

24. Pawarode A, Fine D, Thuluvath P. Independent risk factors and natural history of renal dysfunction in liver transplant recipients. Liver Transpl. 2003;9(7):741-7.

25. Zou X, Jiang K, Puranik A, Jordan K, Tang H, Zhu X, et al. Targeting Murine Mesenchymal Stem Cells to Kidney Injury Molecule-1 Improves Their Therapeutic Efficacy in Chronic Ischemic Kidney Injury. Stem Cells Transl Med. 2018;7(5):394-403. doi: 10.1002/ sctm.17-0186. Epub 2018 Feb 15.

26. Finkenstedt A, Dorn L, Edlinger M, Prokop W, Risch L, Griesmacher $A$, et al. Cystatin $C$ is a strong predictor of survival in patients with cirrhosis: is a cystatin C-based MELD better? Liver Int. 2012; 32(8):1211-6

27. Hei ZQ, Li XY, Shen N, Pang HY, Zhou SL, Guan JQ. Prognostic values of serum cystatin $C$ and beta2 microglobulin, urinary beta2 microglobulin and $\mathrm{N}$-acetyl-beta-D-glucosaminidase in early acute renal failure after liver transplantation. Chin Med J (Engl). 2008; 121(14):1251-6.

28. Sirota J, Walcher A, Faubel S, Jani A, McFann K, Devarajan P, et al. Urine IL-18, NGAL, IL-8 and serum IL-8 are biomarkers of acute kidney injury following liver transplantation. BMC Nephrol. 2013;14:17. doi: 10.1186/1471-2369-14-17

29. Moledina DG, Hall IE, Thiessen-Philbrook H, Reese PP, Weng FL, Schröppel B, et al. Performance of Serum Creatinine and Kidney Injury Biomarkers for Diagnosing Histologic Acute Tubular Injury. Am J Kidney Dis. 2017;70(6):807-816. doi: 10.1053/j.ajkd.2017.06. 031. Epub 2017 Aug 24. 